\title{
Redesigning Teams and Incentives in a Merger: An Experiment with Managers and Students
}

\author{
Claude Montmarquette \\ CIRANO, and University of Montréal, 2020 University Street, Montréal, Québec, Canada H3A 2A5, \\ claude.montmarquette@cirano.qc.ca \\ Jean-Louis Rullière \\ GATE (CNRS, University of Lumiere, Lyon 2, Ecole Normale Supérieure), 93 chemin des Mouilles, \\ 69130 Ecully, France, rulliere@gate.cnrs.fr \\ Marie-Claire Villeval \\ GATE (CNRS, and University of Lumiere, Lyon 2, Ecole Normale Supérieure), 93 chemin des Mouilles, \\ 69130 Ecully, France, and IZA (Institute for the Study of Labor), Bonn, Germany, \\ villeval@gate.cnrs.fr \\ Romain Zeiliger \\ GATE (CNRS, University of Lumiere, Lyon 2, Ecole Normale Supérieure), 93 chemin des Mouilles, \\ 69130 Ecully, France, zeiliger@gate.cnrs.fr
}

\begin{abstract}
A fter a merger, company officials face the challenge of making compensation schemes uniform and of redesigning teams with managers from companies with different incentives, work habits, and recruiting methods. In this paper, we investigate the relationship between executive pay and performance after a merger by dissociating the respective influence of shifts, which occur in both compensation incentives and team composition. The results of a real task experiment conducted with managers within a large pharmaceutical company not only show that changes in compensation incentives affect performance, but also suggest that the sorting effect of incentives in the previous companies impact cooperation and efficiency after the merger. Replicating this experiment with students showed differences in strategy rather than in substance between the two groups of subjects with managers appearing performance driven, while students are more cost driven.

Key words: executive and team-based compensation; subject pool effects; real task experiment; incentives; sorting; mergers

History: Accepted by Linda Argote, organization performance, strategy, and design; received December 10, 2002. This paper was with the authors 10 months for 2 revisions.
\end{abstract}

\section{Introduction}

There is strong evidence of the existence of heterogeneity among the compensation packages applied by firms within the same industry. It is not surprising to find that after a merger, difficulties can arise because of the different compensation policies of the newly merged firms, and that new consolidated policies need to be designed. Furthermore, downsizing and the reorganization of production entail a reshuffling of teams, which affects executives from the companies involved in the merger. To promote social cohesion, mergers usually lead to the harmonization of company statutes, so that all executives are paid according to the same compensation schemes. But within new teams comprised of executives of the merged companies, performance also depends on the willingness of individuals to cooperate with others. This willingness to cooperate may be affected by the heterogeneity of past compensation practices, work habits, and nonmarket interactions. Many pitfalls can hamper an empirical analysis of the relationship between new executive pay packages and executive performance after a merger. The impact of new compensation packages may differ from one employee to another depending on the short-, medium-, and long-term influence of preceding modes of compensation (see Nalbantian and Schotter 1997). Thus, assessing the impact of new compensation schemes on executive performance after a merger requires controlling for the possibility of the long-term impacts of the compensation packages used before the merger. Furthermore, unbiased estimates of the relationship between pay and performance require disentangling the effects of shifts in direct incentives from the effects of the emergence of a new group culture founded on a variety of previous corporate cultures (Kreps 1990). Individuals coming from a variety of previous cultures, with different norms of fairness and social comparison, could be expected to behave differently in the new company. Previous cultures can affect the efficiency of a new unified compensation policy, particularly in the short run. Experimental methods can help 
in circumventing part of these difficulties through the comparison of various treatments in a controlled environment. This point has been successfully made in the context of a merger by Camerer and Weber (2003) who show that performance decreases following the merger of two laboratory firms because of failure in coordination.

In this paper, we design an experiment to analyze the relationship between executive compensation schemes and performance after a merger. Our laboratory experiment was conducted with 36 managers of a large pharmaceutical company created by the recent merger of two companies-one French and one German. It was replicated with 72 students in Lyon and Montréal. Thus, this paper also contributes to the literature on subject pool effects by comparing decisions made by student-subjects and managersubjects. Studies by Dyer et al. (1989) and Carpenter et al. (2003) have underlined differences (risk attitudes, fairness) and similarities (winner's curse) across subject pools. Hannan et al. (2002) show that MBA effort levels are greater than those of undergraduate students, and Fehr and List (2004) observe that CEOs exhibit more trustful behavior. How can one explain that in some games expertise seems to influence behavior whereas in others it has no significant influence? Cooper et al. (1999) emphasize the relationship between context and expertise. Expertise can improve the relative efficiency of manager-subjects in experiments only when they are able to recognize the similarity between the laboratory context and their field experience. Here, we could expect that conducting the experiment within the company context and performing an abstract task, which reproduces characteristics of executive teamwork, should induce a greater level of performance from managers. This paper is also innovative because it examines these effects in a team context with individual and team rewards.

The task required of participants consisted of searching for the highest value of a multiple-peaked function in a 2-dimensional space. This task has a cognitive component because intense concentration is required because of uncertainty and time pressure, and there is a monetary cost linked to the chosen speed of progression. This real-task experiment adds to the limited number of experimental papers (Dickinson 1999, Sillamaa 1999, van Dijk et al. 2001, Falk and Ichino 2003, Gneezy et al. 2003), which study rewards and behavior in a real work setting. Many experiments, which require subjects to choose an effort level not related to a real effort confirm that monetary incentives do matter, but assume the equivalence between intention of contribution and effort, and between disutility of effort and money. Our experiment also differs from naturally occurring experiments (e.g., Lazear 2000) or field experiments (Erev et al. 1993) in which subjects perform a task in a real work environment. In this study, manager-subjects undertake tasks, which reproduce aspects of a manager's job under a familiar structure of incentives. They make their decisions in an artificial environment of anonymous interactions, according to instructions using neutral wording and without field referents.

Our design involves two parts: (1) teams are homogeneous and are paid according to the rules in effect before the merger of the pharmaceutical firms and (2) the teams remain homogeneous or are formed randomly with participants originating from each of the two merged companies or from both schools; all are paid according to the rules in use after the merger. These treatments are aimed at disentangling the effect of the shift in team composition and the impact of the shift in incentive schemes.

Our main results indicate that there is a pure effect on performance of the shift in incentives after the merger. They show that the past does matter in as much as some managers reduce their effort when they are potentially mixed with managers from the other incoming firm. This may be the result of the sorting effect of previous incentives schemes: Paying executives under different rules has probably contributed to the creation of attitudes toward cooperation in teams. Lastly, we find evidence that manager-subjects and student-subjects differ more in strategy than in substance, with managers being oriented more to the maximization of performance, while students focus more on cost minimization. In $\S 2$, the design of the experiment is outlined and $\S 3$ presents the experimental procedures. The empirical results are discussed in $\S 4$. In $\S 5$, we conclude.

\section{Experimental Design}

The experiment consists of two parts. In the first part it reconstitutes the pay structure of the firms before they merge and, in the second part, the pay structure prevailing in the merger. The nature of the task to be performed during the experiment remains the same, thus allowing an analysis of the consequences on performance of both changes in the payment structure and of team composition. In this section, we present the design of the task, the structure of payment schemes, the treatments, and information conditions.

\subsection{Task Design and Behavioral Heuristics}

One original aspect of this experiment lies in the design of a task, which mimics some aspects of the content of a manager's job (concentration, variability, adjustment of means to targets, and ability to cope with uncertainty under time pressure). The challenge was to be able to discriminate the impact of a cognitive effort on the outcome from that of ability. The task consists of searching for the highest value of 
an increasing function built in a 2-dimensional space defined vertically by height $(H)$ and horizontally by distance $(D)$ from the origin, with $H \in[0,100]$, $D \in[0,300]$ and with $H^{\operatorname{Max}}=f(D)$. It is common knowledge that the curve can have single or multiple peaks. When the period starts, the box in which the curve will appear is fully black. During a one-minute period, the subject progressively uncovers the curve on his computer screen starting at the origin, by clicking a button. ${ }^{1}$ The subject progresses by discrete steps on the horizontal axis. She can stop her progression at any moment. The outcome achieved by a subject in a period is given by the maximum height reached on the curve, which depends notably on the number of moves.

The monetary cost associated with the task is represented by the choice of the speed of progression, i.e., the work pace. Two buttons are available to the subjects: (1) a "one-step button" used to uncover the curves at a regular speed and is cost free and (2) a "two-step button" that doubles the speed but which costs 0.4 point. The subject can switch speeds at will. This design allows a control over the subject's task costs and makes possible an analysis of efficiency. It is impossible to reach maximum height during the one-minute period allowed by using the regular speed only, with the exception of 1 of the 13 randomly occurring curves. The subjects do not know this information. A subject's outcome depends on the use of two-step moves and on chance, because there is uncertainty about the distances from the origins to the peaks. Therefore, the cognitive dimension of the task relates to the uncertainty about the shape of the curve, to time pressure, and to the decision to use the oneor two-step button. Unlike the real-task experiment of van Dijk et al. (2001), ours involves no algorithm enabling the discovery of a peak at minimum cost while under time constraints; no one benefits from previous learning. At each point on the curve, the subject cannot infer from the already uncovered part of the curve the slope of the curve at the next point. The subject cannot discover one single algorithm but must build heuristic grounded on a continuous tradeoff between the speed to choose and the time left. For example, if a subject has already used many extra cost moves and the curve remains flat, paying each additional two-step move requires a continuous trade-off between its marginal cost and its expected marginal revenue. Compared to a traditional experiment where effort values are chosen from a payoff table with a unique trade-off between cost and outcome, our task involves an unknown optimal way of reaching the maximum height at a minimum cost because subjects

${ }^{1}$ The experiment was computerized using the REGATE program. All curves used in this experiment and the instructions are available at http://platypus.gate.cnrs.fr/groupware/g_gate/Raventis.htm. do not know the locations of peaks. The property of duality is not checked: The minimization of cost does not correspond to the maximization of the objective. The multiplicity of heuristics and uncertainty concerning the location of peaks prevent any theoretical prediction. ${ }^{2}$

In short, there are two aspects to the task chosen in this experiment: the decision to use the costless or costly way to progress and the speed of progression, where both can be measured. The cognitive problem of how to reach a higher altitude cannot be measured, however. For the rest of this paper, the altitude reached is the observable outcome of the cognitive effort. This output and the cost of progression are the variables to be explained.

\subsection{Payment Schemes}

Another point of originality lies in the design of compensation schemes that combine fixed pay and performance pay, the latter involving both individual and team incentives. The game involves teams consisting of three subjects who have to uncover the same curve. Subjects are not allowed to communicate with teammates and are not informed of their simultaneous progression. Individual earnings from a task in a given period are calculated from the sum of three elements whose amount and relative proportion depend on the stage of the game and the treatment. Specifically, earnings are defined as: $\pi_{\alpha i}=F_{\alpha}+I_{\alpha}+T_{\alpha}$, with $\alpha=\{X, Y\} . X$ and $Y$ correspond to the two firms before they merge, and for simplicity, $X$ and $Y$ subjects keep their respective labels after the merger to track their origin. $F_{\alpha}$ is a fixed wage earned by subject $i$ when her individual outcome reaches a first threshold, $H_{1}^{\min }$, defined by the height reached. This threshold can always be achieved with no cost steps in the time allowed, but this information is not given to subjects. An employer would consider a distance achieved below this benchmark as professional misconduct. $I_{\alpha}$ is an individual bonus earned if $i$ 's outcome reaches a second threshold, $H_{2}^{\min }$ with $H_{2}^{\min }>H_{1}^{\text {min }} . T_{\alpha}$ is a team reward obtained when the sum of individual outcomes by the team of three subjects reaches a third threshold, $H_{3}^{\text {min }}$ with $H_{3}^{\min }>3 H_{2}^{\min }>3 H_{1}^{\min }$. A subject may earn this reward even though she does not reach an output level greater than the level giving her the fixed wage or the individual bonus. This creates an incentive to free ride.

At each repetition of the game, a new curve is randomly drawn whose shape determines the extent of uncertainty faced by the subjects. The analysis of per-

\footnotetext{
${ }^{2}$ Once the curve is known, it is possible to find the best strategy to reach the highest peak at the lowest cost. A referee's suggestion to compare the subject's behavior with this optimal strategy appears worthwhile to pursue to study the learning process and the cognitive effort of the participants.
} 
Payment Schemes in ECU

\begin{tabular}{|c|c|c|c|c|c|}
\hline \multirow[b]{2}{*}{$\begin{array}{l}\text { Group composition } \\
\text { height reached }\end{array}$} & \multicolumn{2}{|c|}{ Part 1-All treatments } & \multicolumn{2}{|c|}{ Part 2-Fixed treatment } & \multirow[b]{2}{*}{$\begin{array}{l}\text { Part 2-Mixed treatment } \\
\text { Teams of } X \text { and } Y \text { subjects }\end{array}$} \\
\hline & $\begin{array}{l}\text { Teams of } \\
X \text { subjects }\end{array}$ & $\begin{array}{l}\text { Teams of } \\
Y \text { subjects }\end{array}$ & $\begin{array}{l}\text { Teams of } \\
X \text { subjects }\end{array}$ & $\begin{array}{l}\text { Teams of } \\
Y \text { subjects }\end{array}$ & \\
\hline$H_{i}<40$ & 0 & 0 & 0 & 0 & 0 \\
\hline $40 \leq H_{i}<60$ & 40 & 60 & 60 & 60 & 60 \\
\hline $60 \leq H_{i} \leq 100$ & 60 & 60 & 80 & 80 & 80 \\
\hline$\sum_{1}^{3} H_{i} \geq 240$ & 100 & 100 & 120 & 120 & 120 \\
\hline
\end{tabular}

Note. For the teams of $X$ subjects, the table should be read as follows. In the first part of the experiment, if an $X$ subject realizes an outcome lower than the first threshold of 40 , she receives no payoff. If she reaches a height between the first $(40)$ and the second threshold $(60)$, she receives only a fixed wage of $40\left(F_{X}\right)$. If the subject reaches the second threshold, she receives a payoff of 60 , consisting of the sum of the fixed wage and the individual bonus $\left(F_{X}+I_{X}\right)$. If the subject's team reaches a cumulated height of 240 , the subject receives a payoff of 100 , corresponding to the sum of the fixed wage, the individual bonus and the team reward $\left(F_{X}+I_{X}+T_{X}\right)$. Part 2 and the teams of $Y$ subjects should be interpreted in a similar manner.

formance and costs must control for the degree of difficulty of the curves. Because of the structure of the compensation package, the difficulty of a curve depends on the location of the various thresholds. An index of difficulty is calculated as: $d=\left(D_{1}\right)^{2}+$ $\left(D_{2}-D_{1}\right)^{2}+\left(D_{3}-D_{2}\right)$, with $D_{1}$ being the abscissa at the origin of the first threshold; $D_{2}$, the abscissa of the second threshold; and $D_{3}$, the abscissa of the maximum height. The more distant the first threshold is from the origin and the greater the distance between the first and second thresholds, the more difficult it becomes to reach additional rewards.

\subsection{Experimental Treatments and Information Conditions}

The experiment aims at identifying the separate influences of changes in incentives and in team composition. To measure the impact of changes in payment schemes, a session was designed having 2 parts of 10 periods each, with a random order of presentation of 13 payoff curves. In the first part, used as a benchmark, we reproduce initial payment schemes that were used before the merger; in the second part, the payment scheme in use after the merger is applied. In the first part, we team $X$ and $Y$ subjects separately, each playing under the payment scheme used in their initial company. Members of $X$ teams may receive a fixed wage, an individual bonus, and a team reward. Earnings for members of $Y$ teams are derived from a fixed wage and a team reward only (see Table 1). The proportion of potential total earnings from the fixed wage is higher for $Y$ subjects than for $X$ subjects, but the same performance is required from all subjects to trigger their fixed payment and team reward. In the second part of the session, the payment scheme is the one used after the merger and is the same for all subjects. It includes a fixed wage, an individual bonus, and a team reward. Compared to the first part, $Y$ subjects may now receive an individual bonus and $X$ subjects have seen an increase in their fixed wage. In avoiding a decrease in the absolute level of any pay component of earnings between the two parts of the session, the maximum earnings all subjects can obtain is now 120 experimental currency unit (ECU) instead of 100 .

To measure the impact of team composition on performance, a new matching treatment was introduced in two sessions. This treatment is referred as the mixed treatment, where teams of three randomly associated subjects are formed potentially involving both $X$ and $Y$ subjects. The fixed treatment used in the first part of all sessions, where $X$ and $Y$ subjects are teamed exclusively with $X$ and $Y$ subjects, respectively, is maintained in the second part in one session. The fixed treatment serves as a benchmark against which the effect of team composition after the merger is tested. A stranger-matching protocol is always used. In the second part of the session, only team composition is changed and not the size of the teams. ${ }^{3}$

All subjects, except those playing the fixed treatment, knew of the existence of two categories of subjects in equal numbers in the room, but they were kept unaware of the meaning of labels $X$ and $Y$. Subjects learned their own identity by reading the instruction sheet and were notified that they would keep the same identity throughout the session. The instruction sheet for the first 10 periods mentioned that they were matched with two other subjects belonging to the same category as themselves and that the composition of groups would, within the same category, change with each new period. They were informed that they would never know the identity and the payoff of their successive teammates. Subjects knew the description of the task to be performed and the payoff structure applicable to their category. The participants were not given information about the payoff structure of the other category of subjects, were aware that the

\footnotetext{
${ }^{3}$ The negative impact on efficiency of the increasing size of teams in a merger has been documented by Camerer and Knez (1994).
} 
same task was to be achieved by the three members of their group, and had no current information about the progression of their teammates on the curve. During play, their screen indicates the time currently left, the cumulated cost of their two-step decisions, and the height of the different thresholds reached. At the end of each period, a historic table gave each subject feedback on their own outcome, the outcome (cumulative height) achieved by their team, total cost, amount of compensation obtained, by source, and total payoff net of costs.

In the second part of the session, subjects were informed about the payoff structure of the first part for each of the two categories. The new payoff structure common to all subjects was explained. In one session, an equal number of subjects from both categories continued to play under the fixed treatment for periods 11-20. In two sessions, subjects drawn in equal numbers from $X$ and $Y$ were informed that during the remaining 10 periods, they could be matched randomly with members of the other category. ${ }^{4}$

\section{Experimental Procedures}

The experiment was first conducted with managers of the pharmaceutical company about two years after the merger and it was later replicated with students in engineering and business from Lyon and Montréal.

Thirty-six volunteer executives were recruited by e-mail with average annual earnings of EUR 69,000, consisting of 18 managers from each incoming firm. To limit uncontrolled peer-group effects, sessions were designed such that the participants represented a large diversity of departments and came from different geographical locations. ${ }^{5}$ The experiment was conducted at the company headquarters in Paris on the same day to limit the dissemination of information. In the first 2 sessions, 12 participants $(6 X, 6 Y)$ were subjected to the mixed-treatment protocol. In the third session, 12 participants played under the fixed-treatment protocol. On average, a session lasted 75 minutes including practice periods. Transactions were conducted in ECUs, with ECU convertible to euros at the rate $150 \mathrm{ECU}=\mathrm{EUR}$ 4.5. A showup fee of 8 euros was added. On average, a subject earned EUR 51.45 (S.D. = 3.75). Subjects were paid a few days later with vouchers.

Upon arrival, each manager had to register and was invited to draw a ticket from an envelope to assign him or her a computer. In fact, company-specific

\footnotetext{
${ }^{4}$ It would have been interesting to test whether belonging to the majority category or the minority category of a team would influence individual behavior within teams. However, this would have required collecting a far greater number of observations. For this reason, we did not inform subjects about the detailed composition of their teams.

${ }^{5}$ Overall, participants came from 4 different sites and 22 departments.
}

envelopes were presented to subjects according to their originating company, but subjects were unaware of this allocation rule. Subjects discovered a set of written instructions for the first part of the session under their keyboard. As the payment schemes differed among $X$ and $Y$ participants, the experimenter did not read the instructions aloud. ${ }^{6}$ Instructions were phrased in neutral terms with queries answered in private. Three practice rounds were run before the experiment began. At the end of the first part, the game stopped and further instructions for the second part were distributed, without any questions allowed.

This experiment was replicated with 72 studentsubjects, in the experimental laboratories of GATELyon and at the LUBC3E-CIRANO in Montréal. Three sessions were organized over 2 days. The two mixed-treatment sessions were played online, the REGATE software enabling the online reshuffling of groups between Montréal and Lyon subjects. During each mixed-treatment session, 12 French subjects and 12 Canadian subjects interacted as subjects $X$ and $Y$ without being informed that they were playing with subjects abroad. The third session (fixed treatment) involved 12 students at each location. Sessions were conducted under the same conditions as sessions with manager-subjects, except that the ECU were convertible at the rate $150 \mathrm{ECU}=\mathrm{EUR} 1$ for the French subjects and at the rate $150 \mathrm{ECU}=\mathrm{C} \$ 1.5$ for the Canadian subjects. A show-up fee of EUR 4 or C $\$ 6$ was added. On average, a subject earned EUR 12.89 (S.D. = EUR 0.80). Subjects were immediately paid in cash in a separate room.

\section{Experimental Results}

\subsection{Overall Statistics}

In Figures 1-3, we present histograms of average output achieved, average rate of free riders and average return to output level plotted against treatment, subject pool, and the group of periods (1-10 or 11-20). Figure 1 shows that the reaction to incentives differs according to the subject pools: the average output level is higher for managers than for students throughout the experiment. For the managers, output increases in the second part of the experiment under the merged company's compensation package. This increase is particularly noticeable in the fixedtreatment sessions, where the only change introduced relates to the increase in monetary incentives; when both incentives and team composition are changed,

\footnotetext{
${ }^{6}$ Reading instructions aloud guarantees that rules are common knowledge. However, the section of instructions related to different payment schemes of the $X$ and $Y$ subjects must remain unknown until the end of the first part of the session. Reading aloud only other sections of the instructions would have focused undue attention on the question of compensation.
} 
Figure 1 Average Output by Subject Pool and by Treatment

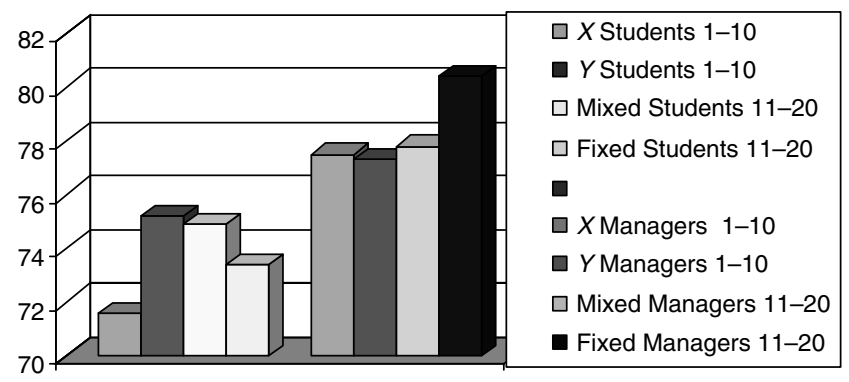

Figure 2 Average Proportion of Free Riders by Subject Pool and by Treatment

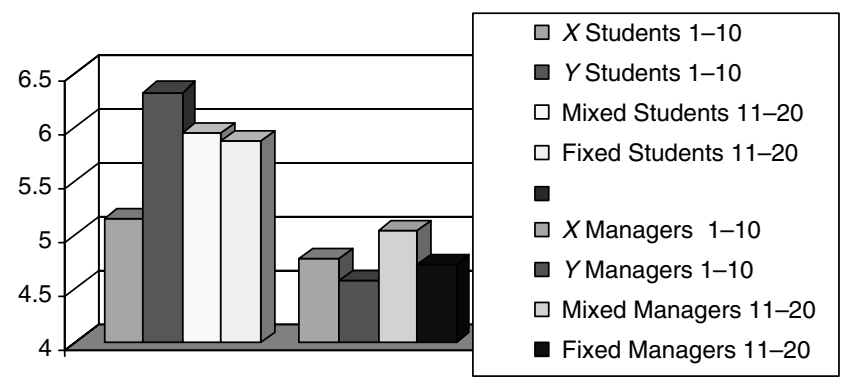

Figure 3 Average Return to Output by Subject Pool and by Treatment

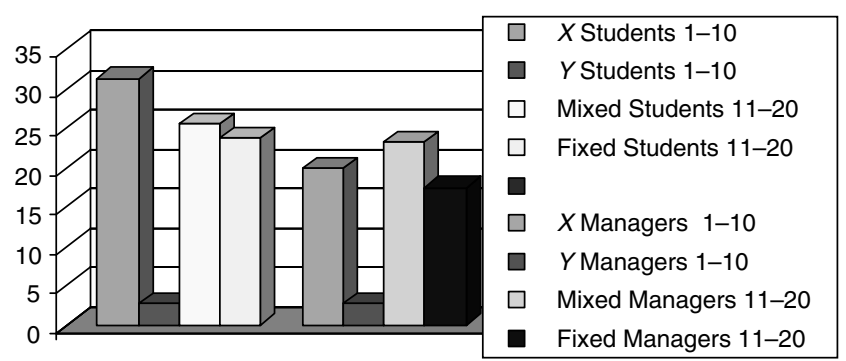

Note. The average indices of difficulty of the curves are the following: In the student sessions, periods 1-10: 22,949; periods 11-20 in the mixed treatment: 24,047 ; periods $11-20$ in the fixed sessions: 21,635 . In the managersessions, periods 1-10: 22,949; periods $11-20$ in the mixed treatment: 22,339; periods $11-20$ in the fixed sessions: 21,635 .

the increase in managers' average output is not significant.

These different output levels may be related to differences in the proportion of free riders in the two pools. Because of the team reward in the payment scheme, our experiment can be connected with the voluntary contributions mechanism (VCM) in public good games. It is interesting to verify whether the coexistence of a fixed wage, an individual bonus, and a team reward in the same compensation scheme changes the behavior toward cooperation as typically observed in these games. Transposing the strict definition of free riding in the VCM to our experiment means that a free rider is defined here in the following way. In part $1, X$ is qualified as a free rider in period $t$ when her output is $\leq 60$, i.e., when the subject does not contribute to the outcome beyond the level that triggers her individual bonus. The same condi- tion applies for part 2. In part $1, Y$ is qualified as a free rider when her output in period $t$ is $\leq 40$, i.e., when she does not contribute to the team outcome beyond the level that secures her fixed wage and, in part 2, when her output level is $\leq 60$. In a typical public-good game, whereas each subject has a dominant strategy not to contribute, the level of contribution is initially positive and declines with repetition (see Ledyard 1995). Figure 2 shows the rate of free riders by subject pool and by treatment for parts 1 and 2 . It indicates that there is a low proportion of free riders compared to traditional results of public good games, probably because of both the coexistence of individual and collective payments and the impossibility of calculating the marginal per capita return of investing in the team outcome. ${ }^{7}$ The proportion of free riders is lower among managers than among students in both parts. $^{8}$ This proportion is lower when the threshold that triggers the greater individual reward is low. It increases in part 2 in both subject pools, particularly in the mixed sessions, and this cannot be explained by a restart effect at the 11th period of the game as will be shown later. This is in line with the declining cooperation over time usually found in publicgood experiments, and could be compared with the differences observed between stranger- and partnermatching protocols (see Keser and van Winden 2000). Figure 3 displays the average return to output level, i.e., the average output achieved divided by the average cost level. On average, managers achieve a lower level of efficiency than students. The former perform a greater output but at a higher cost. In part 2, the average level of efficiency rises slightly in both subject pools.

However, overall statistics are uncontrolled for time, difficulty of the curves and individual effects. Regression analyses controlling for these dimensions are thus required to identify the determinants of two endogenous variables: output levels and costs. In Table 2, we present the definition and descriptive statistics of variables used in these regressions.

Exogenous variables are the period, category of subjects, mode of compensation, composition of groups, and an index of difficulty for each curve. The "lagged output achieved of the other group members" variable assesses whether subjects modulate their outputs to what their teammates did in the previous period. Interaction variables involving the $Y$ subjects are created to test whether $X$ and $Y$

${ }^{7}$ This is evocative of the result found by Charness et al. (2004), that in a gift-exchange game, there is less reciprocity when the payoffs for different actions are made clearer.

${ }^{8}$ The observation that managers free ride less than students is reminiscent of the Hannan et al. (2002) result that MBA students (which are more like managers) do more gift exchange than other students. 
Table 2

Variables and Descriptive Statistics

\begin{tabular}{|c|c|c|c|}
\hline \multirow[b]{2}{*}{ Variable } & \multirow[b]{2}{*}{ Definition } & \multicolumn{2}{|c|}{ Mean (standard deviation) } \\
\hline & & Managers & Students \\
\hline \multicolumn{4}{|l|}{ Endogenous variables } \\
\hline Output achieved & Output levels reached by the subjects & $\begin{array}{c}78.04 \\
(20.96)\end{array}$ & $\begin{array}{l}73.9 \\
(20.680)\end{array}$ \\
\hline Cost & Costs of outputs supported by the subjects & $\begin{array}{c}16.60 \\
(13.19)\end{array}$ & $\begin{array}{c}12.75 \\
(11.39)\end{array}$ \\
\hline \multicolumn{4}{|l|}{ Exogenous variables } \\
\hline 1st period effect & $\begin{array}{l}1 \text { if the } 1 \text { st period, } \\
0 \text { otherwise }\end{array}$ & $\begin{array}{l}0.05 \\
0.95\end{array}$ & $\begin{array}{l}0.05 \\
0.95\end{array}$ \\
\hline 11th period effect & $\begin{array}{l}1 \text { if the } 11 \text { th period, } \\
0 \text { otherwise }\end{array}$ & $\begin{array}{l}0.05 \\
0.95\end{array}$ & $\begin{array}{l}0.05 \\
0.95\end{array}$ \\
\hline$Y$ subject & $\begin{array}{l}1 \text { if the individual is a } Y \text { subject, } \\
0 \text { otherwise ( } X \text { subject) }\end{array}$ & $\begin{array}{l}0.50 \\
0.50\end{array}$ & $\begin{array}{l}0.50 \\
0.50\end{array}$ \\
\hline Mixed session & $\begin{array}{l}1 \text { if } X \text { and } Y \text { subjects can interact, } \\
0 \text { otherwise }\end{array}$ & $\begin{array}{l}0.33 \\
0.67\end{array}$ & $\begin{array}{l}0.33 \\
0.67\end{array}$ \\
\hline Mixed session and $Y$ subject & $\begin{array}{l}1 \text { if } Y \text { is involved in a mixed session, } \\
0 \text { otherwise }\end{array}$ & $\begin{array}{l}0.16 \\
0.84\end{array}$ & $\begin{array}{l}0.16 \\
0.84\end{array}$ \\
\hline Mode of compensation in part 1 & $\begin{array}{l}1 \text { if part } 1 \text { (periods } 1-10 \text { ), } \\
0 \text { if part } 2 \text { (periods } 11-20 \text { ) }\end{array}$ & $\begin{array}{l}0.50 \\
0.50\end{array}$ & $\begin{array}{l}0.50 \\
0.50\end{array}$ \\
\hline $\begin{array}{l}\text { Mode of compensation in part } 1 \\
\text { and } Y \text { subject }\end{array}$ & $\begin{array}{l}1 \text { if a } Y \text { subject in part } 1, \\
0 \text { otherwise }\end{array}$ & $\begin{array}{l}0.25 \\
0.25\end{array}$ & $\begin{array}{l}0.25 \\
0.25\end{array}$ \\
\hline $\begin{array}{l}\text { Lagged output levels of the } \\
\text { other members of the group }\end{array}$ & $\begin{array}{l}\text { Output of the other members of the group } \\
\text { in the preceding period }\end{array}$ & $\begin{array}{l}154.88^{*} \\
(32.07)\end{array}$ & $\begin{array}{l}146.49^{*} \\
(34.67)\end{array}$ \\
\hline $\begin{array}{l}\text { Lagged output levels of the other } \\
\text { members of the group and } Y\end{array}$ & $\begin{array}{l}\text { Output of the other members of the group } \\
\text { in the preceding period and } Y\end{array}$ & $\begin{array}{l}155.15^{* *} \\
(32.37)\end{array}$ & $\begin{array}{l}147.58^{* *} \\
(35.04)\end{array}$ \\
\hline Index of difficulty & Index of difficulty of the curve/100 & $\begin{array}{c}225.27 \\
(143.10)\end{array}$ & $\begin{array}{c}230.96 \\
(143.36)\end{array}$ \\
\hline Index of difficulty squared & Index of difficulty squared & $\begin{array}{c}71,798.18 \\
(72,847.8)\end{array}$ & $\begin{array}{c}73,882.02 \\
(72,466.04)\end{array}$ \\
\hline Index of difficulty and period & Interaction of index of difficulty and period & $\begin{array}{c}2,498.6 \\
(2,482.7)\end{array}$ & $\begin{array}{c}2,581.17 \\
(2,512.12)\end{array}$ \\
\hline Period & Periods 1-20 & 10.5 & 10.5 \\
\hline Logarithm of period & Logarithm of period & 2.12 & 2.12 \\
\hline
\end{tabular}

*1st and 11 th periods excluded.

**For $Y$ subjects only and 1st and 11th periods excluded.

subjects behave differently. Coefficient estimates of the variable "mode of compensation in part 1 " report the decisions of the $X$ subjects in part 1 relative to their decisions under the mode of compensation in part 2 (element of the constant term). With the coefficients of the "mixed session" variable, we further distinguish the decisions of the $X$ subjects in the mixed sessions in part 2 relative to the fixed sessions in part 2. The decisions of the $Y$ subjects in part 1 are the sum of the coefficients of the variables " $Y$ subject," "mode of compensation in part 1, " and " $Y$ subject and mode of compensation in part 1." This last variable is needed as the modes of compensation differ in part 1 between $X$ and $Y$ subjects. Summing up, coefficient estimates of variables " $Y$ subject," "mixed session," and "Y subject and mixed session" give the decisions of $Y$ subjects in the mixed sessions in part 2 . The coefficient of the "Y subject" variable shows the decisions of "Y subjects" in the fixed sessions in part 2.
The index of difficulty and the period variables enter regressions with interacting variables and nonlinear forms. Instead of including a dummy for each period to control for time effects, we distinguish the variable "logarithm of the period" from the "periods 1-20" variable to separate two potentially adverse effects. While the former may account for elements such as boredom that could push output level down over time, the latter may account for learning of the task that could increase output over time. Lastly, demographic variables such as gender and age were entered to control for their potential impact, but because none proved to be significant, they were subsequently removed.

\subsection{Econometric Results}

Column 1 of Tables 3 and 4 display the results for the students and managers, respectively, on the subjects' output achieved. Column 2 of both tables report the results on the determinants of cost levels. The 
Table 3 Students: Determinants of Output Levels and Costs

\begin{tabular}{|c|c|c|c|c|}
\hline \multirow[b]{2}{*}{ Variable } & \multicolumn{2}{|c|}{$\begin{array}{c}\text { Output } \\
\text { Panel random* }\end{array}$} & \multicolumn{2}{|c|}{$\begin{array}{c}\text { Cost } \\
\text { Panel random** }\end{array}$} \\
\hline & Coefficient & $t$-ratio & Coefficient & $t$-ratio \\
\hline Constant & $135.9^{\mathrm{a}}$ & 26.5 & $11.3^{\mathrm{a}}$ & 4.21 \\
\hline 1st period & $-43.09^{\mathrm{a}}$ & -10.9 & 0.671 & 0.362 \\
\hline 11th period & -4.858 & -1.46 & 0.387 & 0.303 \\
\hline$Y$ subject & 3.045 & 0.858 & -1.9608 & -0.831 \\
\hline Mixed & -0.7015 & -0.324 & -1.322 & -1.24 \\
\hline$Y$ and mixed & 1.908 & 0.625 & 1.912 & 1.27 \\
\hline Mode of compensation in part 1 & -2.740 & -1.02 & $-5.27^{\mathrm{a}}$ & -4.10 \\
\hline$Y$ and mode of compensation in part 1 & 2.896 & 1.14 & 1.410 & 1.14 \\
\hline Lagged output levels of other group members & -0.01724 & -1.17 & $0.02892^{\mathrm{a}}$ & 4.18 \\
\hline$Y$ and lagged output levels of other group members & -0.01738 & -1.23 & $-0.01186^{b}$ & -1.79 \\
\hline Index of difficulty & $-0.3076^{\mathrm{a}}$ & -24.2 & $0.03958^{\mathrm{a}}$ & 6.63 \\
\hline Index of difficulty squared & $0.0004981^{a}$ & 21.6 & $-0.00003859^{\mathrm{a}}$ & -3.57 \\
\hline Index of difficulty and period & $0.001846^{\mathrm{a}}$ & 2.73 & $0.0007233^{\mathrm{a}}$ & 2.28 \\
\hline Periods $1-20$ & $1.0409^{\mathrm{a}}$ & 2.50 & $-0.6201^{\mathrm{a}}$ & -3.17 \\
\hline Logarithm of period & $-18.20^{\mathrm{a}}$ & -7.35 & 0.4660 & 0.968 \\
\hline Adjusted $R^{2}$ & \multicolumn{2}{|c|}{0.3569} & \multicolumn{2}{|l|}{0.163} \\
\hline Number of observations & \multicolumn{2}{|c|}{1,440} & \multicolumn{2}{|l|}{1,440} \\
\hline
\end{tabular}

cost incurred corresponds to the number of occasions the two-step button was used to perform the task. All econometric estimates are obtained with a linear one-way random effects model. ${ }^{9}$

For the students considered as a benchmark, few variables significantly influence outputs, except periods and the difficulty of the task. In contrast, the cost levels are influenced by more elements. A change in monetary incentives in the second part of the experiment makes students react by increasing their cost levels but not their output. An increase in the output levels of their teammates during the preceding period incites them to increase their costs, particularly for the $X$ subjects. Lastly, costs decline over time and the relationship between the difficulty of the curves and the costs indicates a reverse $U$-shape.

In contrast, we observe for managers a significant and substantial increase in outputs by both $X$ and $Y$ subjects in part 2 relative to part 1 . The change in incentives after the merger increases outputs by almost 12 points. The change in the composition of teams exerts no significant influence on output. Note, however, the negative but statistically insignificant coefficient of the interaction variable " $Y$ subject and mixed": $Y$ subjects, knowing that they may be inter-

\footnotetext{
${ }^{9}$ Let $E_{i t}$ measure individual $i$ 's output level in period $t$, explained by a vector of observable variables $z_{i t}$, the corresponding parameter vector $\delta$, a random individual component $\eta_{i}$ and a random variable $\varepsilon_{i t}: E_{i t}=z_{i t} \delta+\varepsilon_{i t}+\eta_{i}, i=1, \ldots, n, t=1, \ldots, T$ with the usual assumptions, $\varepsilon_{i t} \sim N(0,1), \eta_{i t} \sim N\left(0, \sigma^{2}\right), \sigma_{\varepsilon \eta}=0$.
}

acting with $X$ subjects, have a tendency to lower their output levels. Despite the stranger-matching protocol, $Y$ subjects are also influenced by the behavior of their teammates in the preceding period: An increase in teammate outputs motivates subjects to increase their own output. It should also be noted that, if we observe a negative first-period effect on output achieved, there is no restart effect at the 11th period. The difficulty of the curves and the number of periods affect outputs in a nonlinear way.

The change in composition of the teams in part 2 has no significant impact on effort decisions, however, it exerts a determinant impact on the cost levels chosen by the subjects. Ceteris paribus, $X$ subjects increase their costs in a mixed session by 6.23 units relative to a fixed session, while $Y$ subjects substantially reduce theirs by 8.38 units when they know they may be teamed with $X$ subjects. ${ }^{10}$ The relationship between the difficulty of the curves and the costs indicates a reverse $U$-shape as with the students. Lastly, there is a positive first-period effect on cost levels, but costs decline more linearly as the experiment evolves.

\subsection{Discussion}

This section will focus on the reactions to the shifts in incentives, in team composition and in the difficulty

\footnotetext{
${ }^{10}$ From the calculation: $[8.06-(8.06+6.23-14.61)]$. The first term is the coefficient of the variable for $Y$ participants in part 2 when groups are fixed. The second term represents the value of the coefficients for $Y$ participants in part 2 when groups are mixed.
} 
Table 4

Managers: Determinants of Output Levels and Costs

\begin{tabular}{|c|c|c|c|c|}
\hline \multirow[b]{2}{*}{ Variable } & \multicolumn{2}{|c|}{$\begin{array}{c}\text { Output } \\
\text { Panel random* }\end{array}$} & \multicolumn{2}{|c|}{$\begin{array}{c}\text { Cost } \\
\text { Panel random** }\end{array}$} \\
\hline & Coefficient & $t$-ratio & Coefficient & $t$-ratio \\
\hline Constant & $125.32^{\mathrm{a}}$ & 13.3 & 6.197 & 1.13 \\
\hline 1st period & -11.80 & -1.59 & $10.25^{\mathrm{a}}$ & 2.42 \\
\hline 11th period & -1.539 & -0.289 & 0.967 & 0.318 \\
\hline$Y$ subject & -3.030 & -0.526 & $8.06^{\mathrm{a}}$ & 2.16 \\
\hline Mixed & 1.500 & 0.414 & $6.23^{\mathrm{a}}$ & 2.83 \\
\hline$Y$ and mixed & -7.194 & -1.41 & $-14.61^{\mathrm{a}}$ & -4.69 \\
\hline Mode of compensation in part 1 & $-11.791^{\mathrm{a}}$ & -2.47 & -1.805 & -0.653 \\
\hline$Y$ and mode of compensation in part 1 & -4.559 & -1.04 & $-10.09^{\mathrm{a}}$ & -3.88 \\
\hline Lagged output levels of other group members & 0.03469 & 1.251 & 0.02427 & 1.53 \\
\hline$Y$ and lagged output levels of other group members & $0.05420^{\mathrm{a}}$ & 2.33 & 0.0195 & 1.37 \\
\hline Index of difficulty & $-0.2132^{\mathrm{a}}$ & -9.41 & $0.05503^{\mathrm{a}}$ & 4.27 \\
\hline Index of difficulty squared & $0.0003381^{\mathrm{a}}$ & 8.37 & $-0.00007559^{\mathrm{a}}$ & -3.29 \\
\hline Index of difficulty and period & $0.003394^{\mathrm{a}}$ & 2.82 & $0.001122^{b}$ & 1.64 \\
\hline Periods $1-20$ & $-1.354^{b}$ & -1.83 & $-1.037^{\mathrm{a}}$ & -2.47 \\
\hline Logarithm of period & $-7.768^{\mathrm{b}}$ & -1.76 & 2.799 & 1.11 \\
\hline Adjusted $R^{2}$ & \multicolumn{2}{|c|}{0.1562} & \multicolumn{2}{|c|}{0.1792} \\
\hline Number of observations & \multicolumn{2}{|l|}{720} & \multicolumn{2}{|l|}{720} \\
\hline $\begin{array}{l}* \text { Linear one-way random effects model. Lagrang } \\
\text { value }=0.000515) \text {. } \\
{ }^{* *} \text { Linear one-way random effects model. Lagrang } \\
\text { value }=0.000000) \text {. } \\
{ }^{\text {a }} \text { Significant at } 5 \% \text { level. } \\
{ }^{b} \text { Significant at } 10 \% \text { level. }\end{array}$ & multiplier tes & $\begin{array}{l}\text { rsus } 0 \\
\text { rsus } 0\end{array}$ & $\begin{array}{l}=12.06(1 \\
=114.41\end{array}$ & obability \\
\hline
\end{tabular}

of the task. Our results provide clear evidence of differences in reactions to a change in monetary incentives between the subject pools. The new compensation scheme in the second part does increase the output levels of $X$ and $Y$ managers. In contrast, the same monetary incentives, controlling for other variables, incite students to increase not their outputs, but their costs to accomplish the different tasks imposed on them. Among managers, $Y$ participants substantially decrease their costs in part 2 . Another difference is the reaction to lagged output levels of the other group members. $Y$ managers increase their outputs if the output levels of their previous teammates increase. Student participants modify their cost levels to the previous output levels of their group members.

These results show that the change in incentives influences both subject pools, with managers appearing to be objective driven, while students are cost driven. This is not that surprising as managers in their professional life are evaluated, remunerated, and promoted by meeting their objectives whatever the cost they must incur to do so (e.g., long working hours). For most students, a large part of the return of their academic effort is from lowering their cost (time devoted to studying a specific matter) to obtain good grades. Note that we were able to observe this kind of result because we used a real costly task. If the student-subjects appear to be more money maximizing than managers in the laboratory, this cannot be attributed to the differences in the opportunity costs to participate, because this was taken into account in the conversion rates.

The changing composition of teams also influences the behavior of subjects. Within the same category ( $X$ or $Y$ ), most subjects are influenced by the behavior of their preceding teammates although teams are reshuffled each period. This might suggest that the subjects refer to their category as a whole. However, the linear one-way effects models recognized that subjects are heterogeneous; our results suggest that subject pools and categories are also heterogeneous. Both $X$ and $Y$ student-subjects are influenced by the output of the subjects they were previously teamed with but they are not influenced by the merging between categories in the second part of the experiment, thus, they do not refer to a specific category but to the whole set of subjects. By contrast, after the merger, the decisions of the category of managers who are more sensitive to the outputs of previous teammates (the $Y$ managers) change behavior knowing that they may be interacting with subjects from the other category; they become less cooperative and they substantially reduce their costs and, to a lesser degree, their outputs. On the contrary, $X$ managers who are not influenced by the behavior of previous teammates react positively to the merging of the categories in 
the same teams. Groups of reference are not the same across subject pools.

In traditional experimental economics literature, reaction to others' decisions is usually characterized by a reciprocity concept (see Fehr and Falk 2002). Our experiment is run with randomly re-matched subjects at each period, so this can also be explained by learning and conditional cooperation: Subjects learn the behavior of other subjects in the same category or in the same room and constantly update their beliefs. But more puzzling is the reaction to the mixing of categories after the merger. Three explanations could be evoked. A first explanation might be that the merger changes preferences by introducing new incentives. This explanation cannot help because we measure the impact of a shift in team composition with other things equal, i.e., we control for changes in incentives. The psychological concept of "in group/out group" (Tajfel et al. 1971) might affect the cooperative behavior of participants. This explanation cannot directly help here because students and managers do not react in the same way. If managers were able to transfer their experience of the merger into the laboratory, whereas the students were not able to do so, this psychological effect might play a role for the managers. But it does not explain why $X$ and $Y$ managers did not react similarly. A more convincing explanation refers to a sorting effect of incentives as documented by Lazear (2000). The incoming companies that merged may differ in recruiting different profiles of managers then developing different cultures because of their various incentive schemes. The preexistence of an individual bonus may contribute to focusing on one's own performance instead on the other's output.

The reaction to the difficulty of the task is similar for managers and students. The $U$-shape curve suggests that more difficult tasks may actually elicit, to some extent, more output. This job-challenge effect is present even in the later stages of the experiment (see the "index of difficulty and period" variable), whereas the logarithm of period exerts a negative effect on the output level (that could be interpreted as boredom or tiredness). This result is consistent with the psychological literature showing that challenging goals lead to higher performance than easy goals (Locke et al. 1981). The job challenge may even be at the initiative of the subject herself (e.g., if she uses targets like reaching the second peak). The relationship between the difficulty of the curves and the costs supported by all participants indicates a reverse $U$-shape. If the task is too difficult, subjects increase their outputs but without resorting to costly two-step moves. This result reinforces our preceding analysis: An increased difficulty does not discourage output under the condition that subjects can save on their costs. Lastly, there is a positive first-period effect on the cost levels (significant for the managers), but costs decline more linearly as the experiment evolves. This is possibly because of a learning effect on the task, on the other's behavior, and on the best moment to use the costly moves. Students appear to learn more than managers as they play the game because they not only decrease their costs, but also increase their outputs (see variable "periods $1-20 ") .{ }^{11}$

\section{Summary and Conclusion}

Executive behavior with respect to performance, motivation, and cooperation is a major element in the success or failure of a merger between companies. Traditionally, economists have suggested looking for an adapted compensation policy to facilitate cooperation within groups of individuals coming from different corporate cultures. The aim of this paper is to check whether a harmonization of compensation packages is sufficient to motivate all managers to cooperate to the same extent. A laboratory experiment has been run involving managers of two large pharmaceutical companies that recently went through a merger. The experimental design introduced various compensation schemes, including an incentive scheme combining individual and team incentives that were implemented in the context of a real task. As in most mergers, these manager-subjects have experienced the redesigning of both compensation schemes and team composition in their newly merged company. The protocol reproduced the pre- and postmerger situation in terms of both compensation and team composition. To complement this experiment, a replication with a subject pool consisting of French and Canadian students was conducted that can serve as a benchmark.

The results show that financial incentives do work in improving output achieved in accordance with standard results (Prendergast 1999). However, the unified incentives are not entirely sufficient to create cooperation among heterogeneous groups, as already experimentally observed (Meidinger et al. 2003). The past matters. In contrast with Nalbantian and Schotter (1997), it matters more in terms of shifting team

\footnotetext{
${ }^{11}$ Despite their different strategies, the similarity of students' and managers' average net payoffs is striking. However, with more output achieved by the managers, the average surplus of a hypothetical firm (height $\times$ a constant - wage costs) is higher when employing manager-subjects rather than student-subjects. The net payoffs are higher under the $Y$ payment scheme than under the $X$ payment scheme before the merger. After the merger, net payoffs are higher when teams, whether composed of students or managers, are homogeneous. In contrast, the hypothetical firm's performance is greater under the $X$ payment scheme before the merger and with mixed teams after the merger.
} 
composition than in terms of incentives, because the change from the two premerger incentive schemes to the unified one increases output in the same proportion. Individuals coming from different corporate cultures, likely with different fairness norms and social-comparison behavior, tend to react differently in the mixed treatment part of our experiment. This may result notably from a sorting effect, attributable to various selection policies in the originating firms: Companies with different incentive policies probably attract different types of managers. This suggests that shifting team composition may limit, at least in the short run, the efficiency of a new unified compensation policy if not taken into account. Merging cultures requires more time than merging incentives and deserves special attention. Our experiment also shows that introducing complex tasks is not necessarily detrimental to performance and cooperation. The concept of job challenge is perhaps more important to soliciting greater effort among employees than is usually suggested in current literature.

Results from the student-subject pool differ in strategy more than in substance, allowing confirmation of the external validity of laboratory experiments. In contrast to the managers, students react to an increase in monetary incentives by accepting more costs to complete a given task rather than increasing their output levels. They are cost driven, whereas managers appear to be objective driven. This means that the managers are also more cooperative and free ride less than the student-subjects. Our results corroborate the interpretation of Cooper et al. (1999) in that when they are able to recognize the similarity between the laboratory context and their field experience, manager-subjects may choose different strategic options than inexperienced subjects. Moreover, it may indicate that if students are more inclined to minimize costs than experts in the laboratory, when one observes the existence of other-regarding preferences in traditional experiments involving studentsubjects, one may deduce that this deviation from the equilibrium is likely more developed in real settings.

\section{Acknowledgments}

The authors are greatly indebted to AVENTIS Pharma for its collaboration in this research. The authors are also grateful to Laurent Volpi, Muriel Prat, and Jean-Benoît GrégoireRousseau for their help in conducting this research, and to David Dickinson and Edward P. Lazear for their useful comments and suggestions. The final version of this paper has benefited from comments and criticisms provided by the associate editor and the referees. This research was supported by the Region of Rhône-Alpes and of the Laboratoire Universitaire Bell (LUBC3E).

\section{References}

Camerer, C. F., M. Knez. 1994. Creating "expectational assets" in the laboratory: "Weakest-link" coordination games. Strategic Management J. 15 101-119.

Camerer, C., R. A. Weber. 2003. Cultural conflict and merger failure: An experimental approach. Management Sci. 49(4) 400-415.

Carpenter, J., S. Burks, E. Verhoogen. 2003. Comparing Students to Workers: The Effects of Social Framing on Behavior in Distribution Games. Mimeo, Middlebury College, Middlebury, VT.

Charness, G., G. Frechette, J. Kagel. 2004. How robust is laboratory gift exchange? Experiment. Econom. 7(2) 189-205.

Cooper, D. J., J. H. Kagel, W. Lo, Q. L. Gu. 1999. Gaming against managers in incentive systems: Experimental results with Chinese students and Chinese managers. Amer. Econom. Rev. 89(4) 781-804.

Dickinson, D. L. 1999. An experimental examination of labor supply and work intensities. J. Labor Econom. 17(4) 608-638.

Dyer, D., J. H. Kagel, D. Levin. 1989. A comparison of naive and experienced bidders in common value offer auctions: A laboratory analysis. Econom. J. 99 108-115.

Erev, I., G. Bornstein, R. Galili. 1993. Constructive intergroup competition as solution to the free rider problem: A field experiment. J. Experiment. Soc. Psych. 29 463-478.

Falk, A., A. Ichino. 2003. Clean evidence on peer pressure. IZA discussion paper 732, Bonn, Germany.

Fehr, E., A. Falk. 2002. Psychological foundations of incentives. Eur. Econom. Rev. 46 687-724.

Fehr, E., J. A. List. 2002. The hidden costs and returns of incentives-Trust and trustworthiness among CEOs. J. Eur. Econom. Assoc. 2(5) Forthcoming.

Gneezy, U., M. Niederle, A. Rustichini. 2003. Performance in competitive environments: Gender differences. Quart. J. Econom. 118 (August) 1049-1074.

Hannan, R. L., J. H. Kagel, D. V. Moser. 2002. Partial gift exchange in an experimental labor market: Impact of subject population differences, productivity differences, and effort requests on behavior. J. Labor Econom. 20(4) 923-951.

Keser, C. F. van Winden. 2000. Conditional cooperation and voluntary contributions to public goods. Scand. J. Econom. 102(1) 23-29.

Kreps, D. 1990. Corporate culture and economic theory. J. Alt, K. Schepsle, eds. Perspective on Positive Political Economy. Cambridge University Press, Cambridge, U.K., 90-143.

Lazear, E. P. 2000. Performance pay and productivity. Amer. Econom. Rev. 90(5) 1346-1361.

Ledyard, J. O. 1995. Public goods: A survey of experimental research. J. H. Kagel, A. E. Roth, eds. Handbook of Experimental Economics. Princeton University Press, Princeton, NJ, 111-194.

Locke, E. A., L. M. Saari, K. N. Shaw, G. P. Latham. 1981. Goal setting and task performance: 1969-1980. Psych. Bull. 90(1) 125-152.

Meidinger, C., J. L. Rulliere, M. C. Villeval. 2003. Does team-based compensation give rise to problems when agents vary in their ability? Experiment. Econom. 6 253-272.

Nalbantian, H. R., A. Schotter. 1997. Productivity under group incentives: An experimental study. Amer. Econom. Rev. 87 314-341.

Prendergast, C. 1999. The provision of incentives in firms. J. Econom. Lit. XXXVII(1) 7-63.

Sillamaa, M. A. 1999. How work effort responds to wage taxation: An experimental test of a zero top marginal tax rate. J. Public Econom. 73 125-134.

Tajfel, H., C. Flament, M. G. Billig, R. P. Bundy. 1971. Social categorization and intergroup behavior. Eur. J. Soc. Psych. 1 147-175.

van Dijk, F., J. Sonnemans, F. van Winden. 2001. Incentive systems in a real effort experiment. Eur. Econom. Rev. 45 187-214. 